\title{
Presynaptic Calcium Is Increased during Normal Synaptic Transmission and Paired-Pulse Facilitation, but Not in Long-Term Potentiation in Area CA1 of Hippocampus
}

\author{
L. G. Wu and P. Saggau \\ Division of Neuroscience, Baylor College of Medicine, Houston, Texas 77030
}

\begin{abstract}
We examined the relationship between presynaptic calcium levels and postsynaptic potentials during normal synaptic transmission, paired-pulse facilitation (PPF), and long-term potentiation (LTP) in CA3-CA1 synapses of hippocampus. By selectively loading the presynaptic terminals with the calcium indicator fura-2, we simultaneously recorded a presynaptic calcium (Ca) transient and the corresponding field EPSP evoked by a single stimulus given to the Schaffer collateral-commissural pathway in guinea pig hippocampal slices. A volume average presynaptic $\mathrm{Ca}$ influx was obtained by taking the first time derivative of the $\mathrm{Ca}$ transient. Our data indicate that the synaptic transmission represented by the initial slope of the field EPSP is approximately proportional to the fourth power of the presynaptic $\mathrm{Ca}$ influx, the volume average $\mathrm{Ca}$ current. Our results in combination with similar findings at the squid giant synapse (Augustine et al., 1985b; Augustine and Charlton, 1986) suggest that the relationship between $\mathrm{Ca}$ influx and transmitter release is well conserved from the molluscan to the mammalian nervous system.
\end{abstract}

A transient increase of the residual Ca level $\left([\mathrm{Ca}]_{\text {res }}\right)$ is generally thought to be the mechanism underlying PPF (Katz and Miledi, 1968; Charlton et al., 1982); however, the relationship between PPF and the presynaptic $[\mathrm{Ca}]_{\text {res }}$ had not been examined before. Our results demonstrate that PPF is approximately linearly related to the $[\mathrm{Ca}]_{\text {res }}$. This finding further supports the residual Ca hypothesis for PPF.

Accumulated evidence from other groups suggests that the presynaptic site contributes to the maintenance of LTP in CA3-CA1 synapses (Bekkers and Stevens, 1990; Malinow and Tsien, 1990); however, our data show that neither an increase of the $\mathrm{Ca}$ transient nor a sustained increase of the $[\mathrm{Ca}]_{\text {res }}$ occurs in the presynaptic terminals during maintenance of LTP. This suggests that the presynaptic mechanism underlying LTP must be downstream to $\mathrm{Ca}$ influx.

IKey words: hippocampus, presynaptic calcium, calcium channel, paired-pulse facilitation, long-term potentiation, synaptic transmission, fura-2, guinea pig, CA1]

\footnotetext{
Received Apr. 12, 1993; revised July 8, 1993; accepted July 20, 1993.

We thank Drs. R. Gray and D. Johnston for kindly allowing us to use some of their equipment, and Drs. O. Delbono, R. Gray, and P. E. Schulz for critical and helpful comments on the manuscript. The computer software for data acquisition and analysis was developed by S. S. Patel

Correspondence should be addressed to Dr. Peter Saggau at the above address. Copyright $(1994$ Society for Neuroscience $0270-6474 / 94 / 140645-10 \$ 05.00 / 0$
}

Presynaptic Ca channels play a key role in synaptic transmission and different forms of synaptic plasticity. So far, most studies have been performed at the squid giant synapse (Llinás et al., 1976, 1981; Augustine et al., 1985a,b) or the neuromuscular junction (Dodge and Rahamimoff, 1967; Kat7 and Miledi, 1968; Delaney et al., 1989). Recently, Regehr and Tank (1991a,b) developed a method for selectively filling mossy fiber terminals with a $\mathrm{Ca}$ indicator by localized perfusion with fura-2 AM in hippocampal slices. This technique allowed them to study the role of residual $\mathrm{Ca}$ in individual mossy fiber terminals following a train of stimulation. We used a different approach to load presynaptic terminals of CA3-CA1 synapses selectively with this $\mathrm{Ca}$ indicator by a localized injection into hippocampal slices. This approach enabled us to record simultaneously a presynaptic $\mathrm{Ca}$ transient from a population of CA3-CAl synapses and the corresponding field EPSP evoked by a single stimulus given to the Schaffer collateral-commissural (SCC) pathway. With this technique, we examined the relationship between presynaptic Ca and the field EPSP during normal synaptic transmission, paired-pulse facilitation (PPF), and long-term potentiation (LTP) in CA3-CA1 synapses of hippocampus.

Early studies reported that the amplitude of the postsynaptic potential has an extremely steep dependence on extracellular $\mathrm{Ca}$ concentration, approximating a third- to fourth-power function in both squid giant synapse (Katz and Miledi, 1970) and neuromuscular junction (Dodge and Rahamimofr, 1967), and a second-power function in hippocampal area CA1 (Dingledine and Somjen, 1981; Mulkeen et al., 1988). Two-electrode voltage-clamp studies of the squid giant synapse have shown that the postsynaptic potential has a relatively linear dependence on the presynaptic Ca current, approximating a first- to secondpower function (Llinás et al., 1976, 1981; Augustine and Eckert, 1984). However, with a three-electrode voltage-clamp technique, which provides a more accurate measurement of presynaptic Ca current, Augustine and colleagues (Augustine et al., 1985b; Augustine and Charlton, 1986) found that the relationship between presynaptic Ca current and postsynaptic potential follows a third-power function in the squid giant synapse. The latter finding suggests that the "cooperative" action of Ca takes place at the step or steps beyond the entry of $\mathrm{Ca}$ into the presynaptic terminals. Until now, the squid giant synapse was the only preparation in which this relationship between presynaptic Ca current and synaptic transmission has been examined. We extended this investigation to the mammalian CNS by means of the above approach (Saggau et al., 1992).

Recent evidence showed that use-dependent short-term syn- 
aptic enhancement, such as augmentation and posttetanic potentiation, depends on the residual intracellular Ca level $\left([\mathrm{Ca}]_{\text {res }}\right)$ in the presynaptic terminals (Connor et al., 1986; Delaney et al., 1989; Delaney and Tank, 1991; Regehr and Tank, 1991b). In addition, PPF is also generally thought to depend on [Ca $]_{\text {res }}$ (Katz and Miledi, 1968; Charlton et al., 1982). However, the relationship between PPF and $[\mathrm{Ca}]_{\text {res }}$ has not been directly examined before, due to the typically low signal-to-noise ratio in optical measurements of the Ca transient evoked by pairedpulse stimulation. With our new technique (Saggau et al., 1992), we found that PPF was approximately linearly related to presynaptic $[\mathrm{Ca}]_{\mathrm{res}}$ in a population of CA3-CA1 synapses of hippocampus.

LTP is generally thought to be involved in learning and memory. Multiple lines of evidence including results of quantal analysis (Bekkers and Stevens, 1990; Malinow and Tsien, 1990; Kullmann and Nicoll, 1992; Liao et al., 1992; Malgaroli and Tsien, 1992) and the demonstration of postsynaptically released presumed retrograde messengers (Korn and Faber, 1991; Fazeli, 1992) strongly suggest that the presynaptic site participates in the maintenance of LTP in area CA1 of the hippocampus. Three mechanisms may underlie a presynaptic maintenance of LTP: (1) an increase of the presynaptic Ca transient during an afferent action potential, such as that which accounts for certain shortterm and long-lasting increases in synaptic strength in the marine snail Aplysia (Kandel and Schwarz, 1982; Byrne et al., 1989); (2) a sustained elevation of [Ca $]_{\text {res }}$ in presynaptic terminals, which may account for posttetanic potentiation (Delaney et al., 1989; Delaney and Tank, 1991; Regehr and Tank, 1991b); and, (3) increased sensitivity of the transmitter-releasing process to the presynaptic Ca transient, such as in mobilization, docking, and fusion of synaptic vesicles, which may account for 5-HTmediated enhancement of transmitter release at crayfish neuromuscular junction (Delaney et al., 1991). Our optical recording technique allowed us to examine these three hypotheses and determine that the former two are unlikely so that the presynaptic maintenance of LTP must be due to enhancement of an effect that is downstream to the presynaptic $\mathrm{Ca}$ influx.

Some of these results have been published in abstract form (Saggau and Wu, 1992; Saggau et al., 1992).

\section{Materials and Methods}

Brain slice and field potential recording. Guinea pigs about 2 months old were anesthetized (methoxyflurane) and quickly decapitated. The brains were immediately removed and $400 \mu \mathrm{m}$ transverse hippocampal brain slices were prepared on a vibrating tissue cutter and stored in artificial cerebrospinal fluid (ACSF) of the following composition (in $\mathrm{mM}$ ): $\mathrm{NaCl}, 124 ; \mathrm{KCl}, 5 ; \mathrm{CaCl}_{2}, 2.5 ; \mathrm{MgCl}_{2}, 1.2 ; \mathrm{NaHCO}_{3}, 22 ;$ D-glucose, 10. The ACSF was temperature controlled at $28-30^{\circ} \mathrm{C}$ and gassed with $95 \% \mathrm{O}_{2}$ and $5 \% \mathrm{CO}_{2}$, maintaining a constant $\mathrm{pH}$ of 7.4 . After a resting period of at least $1 \mathrm{hr}$, the slices were transferred to the recording chamber. Field potentials $(0.6-1 \mathrm{mV})$ were recorded from stratum radiatum with glass microelectrodes $(2 \mathrm{M} \mathrm{NaCl}, 1-5 \mathrm{M} \Omega)$. The recording system (DAS 50, Keithley-Metrabyte) was computer controlled (AT486/25, IBM clone). Before calculating the initial slopes of field EPSPs, all traces of the electrical signals have been corrected for contamination by the presynaptic volleys by subtracting responses obtained during routine application of the glutamate receptor antagonists 6-cyano-7nitroquinoxaline-2,3-dione (CNQX) and aminophosphonovalerate (APV). In addition, this corrected traces demonstrated that the afferent volleys and thus the stimulus intensity remained constant throughout the experiment. Data are expressed as mean \pm SEM.

Loading technique and fluorometric $\mathrm{Ca}$ recording. The $\mathrm{Ca}$ indicator was prepared as follows: $50 \mu \mathrm{g}$ of fura-2 AM (Molecular Probes) was dissolved in $5 \mu$ l of dimethyl sulfoxide (DMSO) containing $10 \%$ pluronic acid, $50 \mu \mathrm{l}$ of ACSF was added, and then the mixture was sonicated for
$1 \mathrm{~min}$. The final solution contained $0.9 \mathrm{~mm}$ fura-2 AM, 10\% DMSO and $1 \%$ pluronic acid. Fura-2 AM solution was prepared each day and used within $1 \mathrm{hr}$. A patch pipette with a tip diameter of about $2 \mu \mathrm{m}$ was filled with this solution, connected to Picospritzer II (General Valve), and positioned in the stratum radiatum (Fig. 1A). The tip of the pipette was lowered until it was $200-250 \mu \mathrm{m}$ below the surface of the slice. A small amount (less than $1 \mu \mathrm{l}$ ) of fura- $2 \mathrm{AM}$ solution was pressure injected into the slice, and then the pipette was withdrawn. Under a fluorescence microscope, we saw a small bright spot that remained in the stratum radiatum throughout the experiment. The membrane-permeant fura- 2 AM locally filled the axons and was then converted by intracellular esterases into the membranc-impermcant form, which diffused to the terminals. About $1 \mathrm{hr}$ after injection, fluorescence emerging from an area with a diameter of about $150 \mu \mathrm{m}$ in stratum radiatum, about 500 $\mu \mathrm{m}$ away from the injection site, was detected by a single photodiode, connected to a low-noise current-to-voltage converter and amplifier. The optical recording system for the $\mathrm{Ca}$ signals includes an inverted microscope (Axiovert 10, Achroplan 50 $\times$ NA 0.9, Zeiss) equipped with a xenon light source, an electromechanic shutter (Uniblitz, Vincent), and a quartz illuminator, as well as matched epifluorescence filter sets for ratio measurements (360:380 $\mathrm{nm}$; Omega). Calibration of Ca signals was obtained by forming the ratio $(R)$ between two continuous recordings (typically $100 \mathrm{msec}$ long, but lasting up to $3 \mathrm{sec}$ ) of fura- 2 fluorescence intensity at two excitation wavelengths with an interval of 5-10 sec (Grynkiewiez, 1985). The fluorescence intensities did not change significantly within this interval. $R_{\min }$ and $R_{\max }$ werc determincd using Ca calibration buffer kit II (Molecular Probes) to which $50 \mu \mathrm{M}$ fura-2 had been added. The background fluorescence of the brain slice was measured before the injection of fura- $2 \mathrm{AM}$ and corrected before computing ratios. A viscosity correction was not made since its exact value is unknown. This could cause an error in calculating the absolute $\mathrm{Ca}$ concentration, but should not interfere with the more important relative $\mathrm{Ca}$ values. The amplitude of the $\mathrm{Ca}$ transient was measured as the difference between the maximal concentration and the resting level. Averaging techniques ( $n \leq 4$; interval, $10 \mathrm{sec}$ ) were used to improve the signal-to-noise ratio. Data are expressed as mean \pm SEM.

\section{Results}

Selective loading of presynaptic and postsynaptic structures With the loading technique described above, the $\mathrm{Ca}$ transients and the field EPSPs evoked by a single orthodromic stimulation of SCC pathway were simultaneously recorded in stratum radiatum. To verify the presynaptic origin of the observed $\mathrm{Ca}$ transients, two controls were performed at the end of each expcriment: (1) bath application of glutamatc antagonists $(10 \mu \mathrm{M}$ CNQX $+50 \mu \mathrm{M}$ APV) abolished the synaptic transmission in the SCC pathway without affecting the presynaptic volley and the corresponding $\mathrm{Ca}$ transient (Fig. $1 B$, left), and (2) antidromic activation of pyramidal cells in the same CAl region resulted in a clear field action potential, but did not cause any detectable Ca transients (Fig. $1 B$, right).

To explore further the selectivity of the loading technique, this method was also applied to the postsynaptic site by injecting a small amount of fura- $2 \mathrm{AM}$ in the alveus, which contains the axons of CAl pyramidal cells (Fig. $2 A$ ). This resulted in a selective loading of CA1 pyramidal neurons with fura-2, as judged by the fluorescence emerging from stratum radiatum (Fig. $2 A$, shaded area) where the apical dendrites of these neurons are located. Controls similar to those of the presynaptic loading were obtained: (1) bath application of glutamate antagonists (10 $\mu \mathrm{M}$ CNQX $+50 \mu \mathrm{M}$ APV) abolished both synaptic transmission and the corresponding $\mathrm{Ca}$ transients evoked by a single orthodromic stimulation of the SCC pathway in the stratum radiatum (Fig. 2B, left), (2) antidromic stimulation of axons of CAl pyramidal neurons in the alveus induced a field action potential and a corresponding $\mathrm{Ca}$ transient (Fig. $2 B$, right), and (3) the onset of the postsynaptic Ca transients evoked by orthodromic 
A
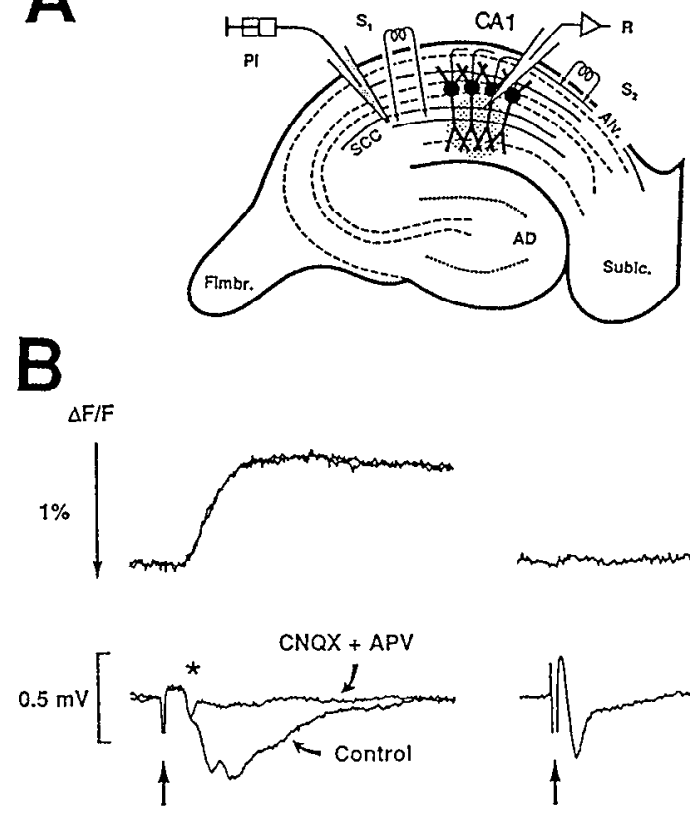

stim. (orthodr.)
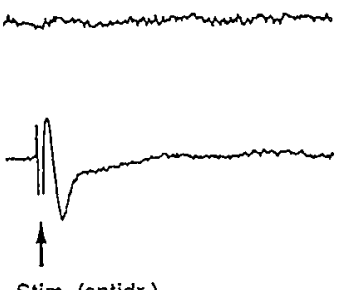

Stim. (antidr.)

Figure 1. Selective presynaptic loading with calcium-sensitive indicator fura-2 AM. $A$, Membrane-permeant calcium indicator (fura-2 AM, $0.9 \mathrm{~mm})$ was pressure injected $(P I)$ under visual control into stratum radiatum, resulting in loading and subsequent diffusion of the indicator via the SCC pathway to presynaptic terminals. About $1 \mathrm{hr}$ after injection, fluorescence from an area with a diameter of about $150 \mu \mathrm{m}$ in stratum radiatum, $500 \mu \mathrm{m}$ away from the injection site, was recorded by a single photodiode (shaded area). The preparation was stimulated (intensity, about $500 \mu \mathrm{A}$ for $200 \mu \mathrm{sec}$; repetition rate, $0.1 \mathrm{~Hz}$ ) by bipolar electrodes $\left(S_{1}, S_{2}\right)$ : orthodromically via the SCC pathway by $S_{1}$, and antidromically via the alveus $\left(A l v\right.$.) by $S_{2}$. An cxtracellular recording clectrode $(R)$ was placed in the center of the shaded area in the area of the CAl apical dendrites. $A D$, area dentata; Fimbr., fimbria; Subic., subiculum. $B$, Calcium signals and the corresponding field potentials. Left, Bath application of glutamate antagonists ( $10 \mu \mathrm{M}$ CNQX $+50 \mu \mathrm{M}$ APV) abolished the field EPSP evoked by an orthodromic (orthodr.) stimulation (Stim.) of the SCC pathway, while the presynaptic volley persisted (see asterisk in lower traces). Synchronous calcium transients did not decrease (upper traces), indicating a presynaptic locus. Right, antidromic (antidr.) activation of pyramidal cells in the same $C A \mathrm{I}$ region resulted in a clear field action potential, but did not cause any detectable calcium transients.

stimulation was examined and was delayed by about $3.5-5 \mathrm{msec}$ compared to the presynaptic Ca transients (Fig. 1B).

\section{Time course of the presynaptic Ca transient and $\mathrm{Ca}$ influx}

The injection of fura-2 AM did not significantly change the slope of the field EPSP, suggesting that fura-2 did not buffer the intracellular $\mathrm{Ca}$ to such an extent as to affect fast synaptic transmission in our experiments. Figure 3 shows the time course of the presynaptic $\mathrm{Ca}$ transient and $\mathrm{Ca}$ influx evoked by a single stimulation of the SCC pathway from a typical experiment. A presynaptic $\mathrm{Ca}$ transient lasted for about 3-5 sec with a halfdecay time of $238 \pm 18 \mathrm{msec}$ (eight slices). Transients of similar time course (half-decay time of 120-300 msec) evoked with single stimuli have been recorded with fura- 2 from the same CA3-CA1 synapses in which synaptic transmission was blocked by CNQX (Saggau and Sheridan, 1989) or by kynurenic acid (Hess and Kuhnt, 1992), and from rat optic nerve (Lev-Ram

A

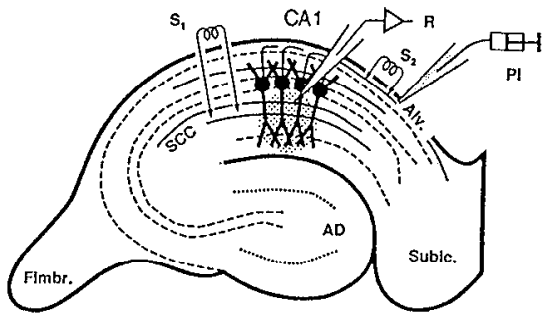

B
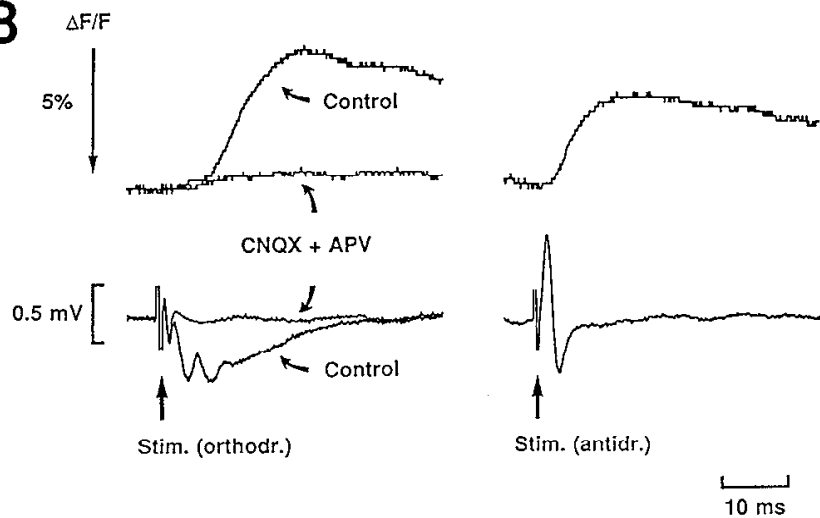

Figure 2. Selective postsynaptic loading with calcium-sensitive indicator. $A$, Fura-2 AM was pressure-injected $(P I)$ under visual control into the alveus $(A l v$.), resulting in loading apical dendrites of CAl pyramidal cells with the indicator. Fluorescence was recorded as before. $B$, Calcium signals and the corresponding field potentials. Left. Bath application of glutamate antagonists (10 $\mu \mathrm{M}$ CNQX $+50 \mu \mathrm{M}$ APV) abolished both the synaptic transmission and the Ca transient. Right, Antidromic activation of pyramidal cells in the same CA1 region resulted in both a field action potential and a Ca transient. For abbreviations, see Figure 1.

and Grinvald, 1987). Since it takes several seconds for the Ca level to return to control following a single stimulation, this suggests that the $\mathrm{Ca}$ removal is much slower than $\mathrm{Ca}$ influx in the presynaptic terminals. Little is known about the endogenous $\mathrm{Ca}$ buffers in the presynaptic terminals. We assume that the fura-2 signal we recorded is proportional to the total amount of $\mathrm{Ca}$ that enters following an action potential. By taking the first derivative of the $\mathrm{Ca}$ transient over time $(d[\mathrm{Ca}] / d t)$, a curve representing the volume average presynaptic $\mathrm{Ca}$ influx was generated (Fig. $3 A$, middle trace; $3 B$, indicated trace). The Ca influx lasted for $15+1 \mathrm{msec}$ ( 10 slices). The peak of $\mathrm{Ca}$ influx was $2.1 \pm 0.2 \mathrm{msec}(10$ slices) earlier than the peak of the corresponding field EPSP. The half-width of the $\mathrm{Ca}$ influx was 5.4 $\pm 0.3 \mathrm{msec}$ (nine slices). The time difference between the onset of $\mathrm{Ca}$ influx and the presynaptic volley was less than $0.5 \mathrm{msec}$. Since the Ca transients were recorded from a circular area with a diameter of $150 \mu \mathrm{m}$, and the velocity of the propagation of action potential in the SCC pathway is about $300 \mu \mathrm{m} / \mathrm{msec}$ (Andersen et al., 1978), we were not able to differentiate a time difference of less than $0.5 \mathrm{msec}$.

\section{Synaptic transmission and the presynaptic Ca transient}

The field EPSP, the presynaptic Ca transient, and the corresponding $\mathrm{Ca}$ influx were investigated during the application of different concentrations of cadmium, a blocker of voltagc-dcpendent $\mathrm{Ca}$ channels (Fig. 4A). Cadmium dose-dependently blocked the presynaptic $\mathrm{Ca}$ transient, $\mathrm{Ca}$ influx, and the corresponding field EPSP (Fig. 4B). The relationship between the normalized amplitudes of $\mathrm{Ca}$ influx $(Z)$ and the presynaptic $\mathrm{Ca}$ 

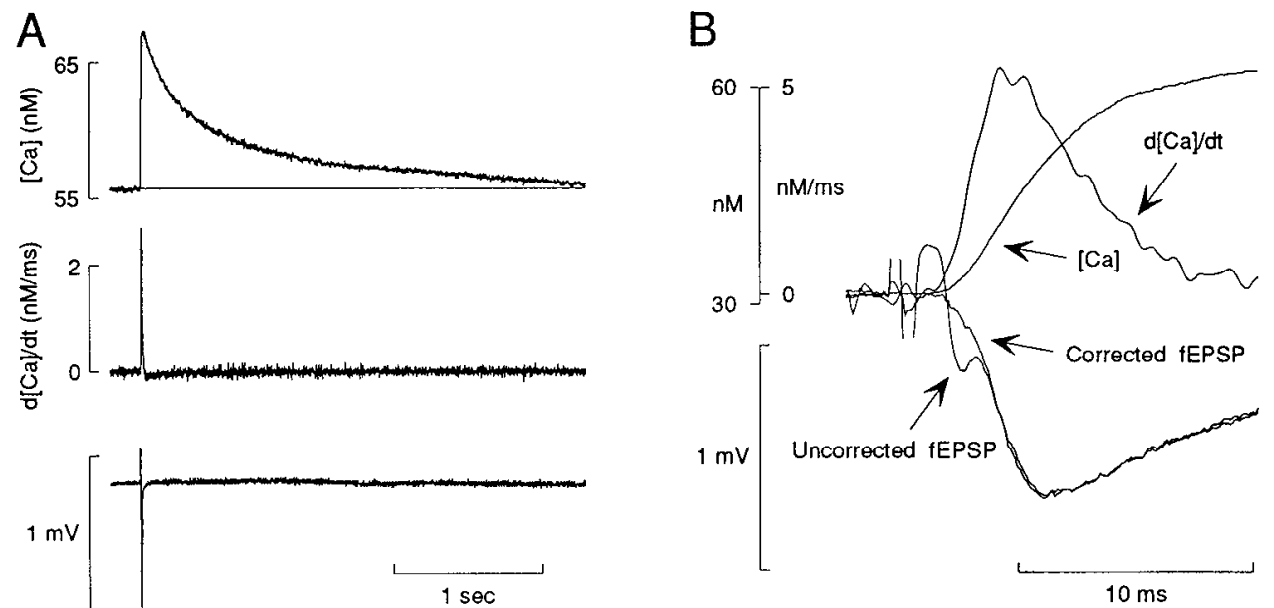

Figure 3. Time course of the presynaptic $\mathrm{Ca}$ transient, $\mathrm{Ca}$ influx, and the corresponding field EPSP from typical experiments. A:Upper trace, A presynaptic $\mathrm{Ca}$ transient evoked by a single stimulus to the SCC pathway. The horizontal line refers to the cytosolic free Ca concentration at rest. Middle trace, A Ca influx obtained from the derivative of the Ca transient over time $(d|\mathrm{Ca}| / d t)$ with a time interval (dt) of $2 \mathrm{msec}$. Lower trace, The corresponding field EPSP. The sampling frequency was $2 \mathrm{kHz}$. B, The presynaptic Ca transient ([Ca]), Ca influx $(d[\mathrm{Ca}] / d t)$, and the corresponding field EPSP $(f E P S P)$ from another slice are plotted with higher time resolution. The corrected field EPSP was computed by subtracting the response in the presence of CNQX and APV, from the uncorrected field EPSP obtained in the presence of normal ACSF. Without the contamination of a presynaptic volley, the initial slope of the field EPSP can be measured more accurately. The sampling frequency was $10 \mathrm{kHz}$. The time interval (dt) for calculating $d[\mathrm{Ca}] / d t$ was $0.4 \mathrm{msec}$.

transients $(X)$ could be well fitted by a linear regression line in each of six slices (Fig. 5A):

$$
Z-a X+b
$$

where the mean of $a$ is $1.01 \pm 0.03$, the mean of $b$ is $0.00 \pm$ 0.03 , and the mean of correlation coefficient $(r)$ is $0.98 \pm 0.01$; thus, Equation 1 can be approximated as

$$
Z=X \text {. }
$$

Consequently, a measurement of the amplitude of a Ca transient is an accurate estimation of the corresponding $\mathrm{Ca}$ influx evoked by a single stimulus to the SCC pathway. Figure $5 A$ shows a typical example from one slice $(r=0.99)$. In contrast, the relationship between the slope of the field EPSP $(Y)$ and the amplitude of the presynaptic $C$ a transient $(X)$ is not linear. It could be fitted by the equation

$$
Y=X^{n},
$$

where the mean of $n$ is $3.6 \pm 0.5$ (six slices), and $x$ and $Y$ are normalized to their baselines (Fig. $5 B, C$ ). The slope of the field EPSP is also related to the amplitude of the $\mathrm{Ca}$ influx by a very similar power function:

$$
Y=Z^{m},
$$

where the mean of $m$ is $3.7 \pm 0.4$ (six slices), and $Z$ and $Y$ are normalized to their baselines (Fig. $5 D, E$ ). Based on this power relationship, a $20 \%$ increase in the presynaptic Ca transient or $\mathrm{Ca}$ influx will increase the field EPSP by about $100 \%$. It was necessary to determine this relationship in order to determine the sensitivity of our measurement before examining whether
Figure 4. Cadmium blocks the presynaptic Ca transient ([Ca]), Ca influx $(d[\mathrm{Ca}] / d t)$, and the corresponding field EPSP $(f E P S P) . A$, Wash-in of 0.1 then $0.3 \mathrm{~mm}$ cadmium reduced the normalized amplitude of the Ca transients, $\mathrm{Ca}$ influx, and the slope of the field EPSPs as shown for a typical experiment. $B$, Sample recordings obtained at the times indicated in $A(a-d)$.
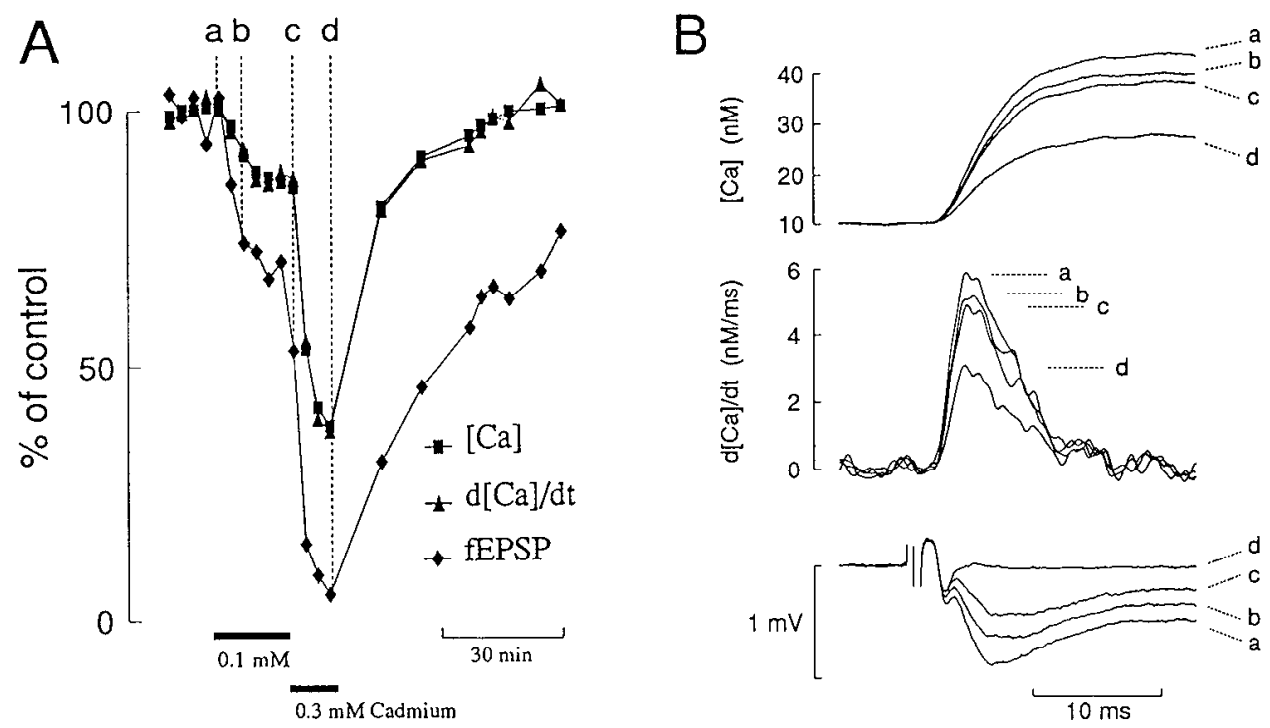


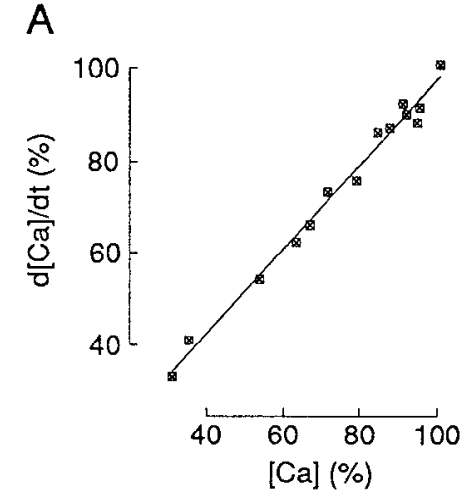

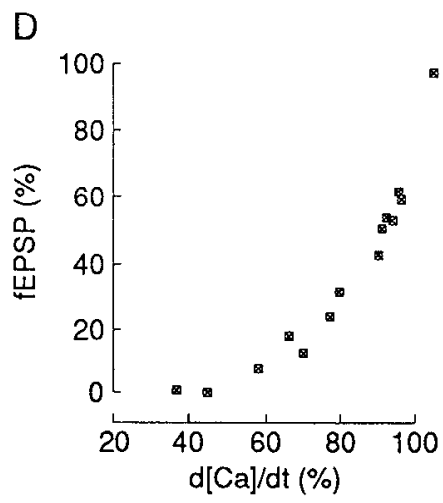

B

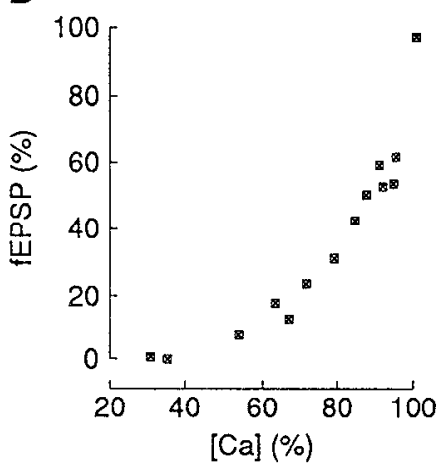

(20)

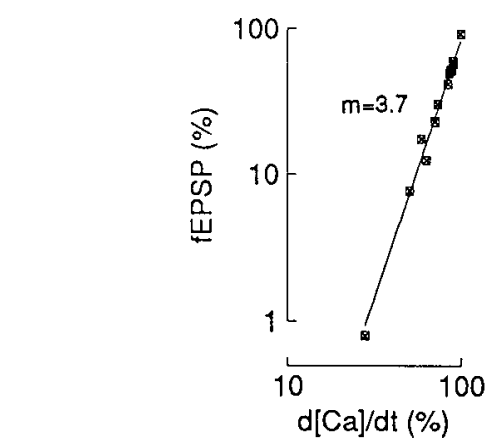

Figure 5. The relationship between presynaptic Ca transient ([Ca]), Ca influx ( $d[\mathrm{Ca}] / d t)$, and the ficld EPSP $(f E P S P)$ from a typical cxperiment determined by applying cadmium to block voltage-dependent $\mathrm{Ca}$ channels. All data have been normalized to baseline. $A$, The relationship between the amplitude of the $\mathrm{Ca}$ influx and the corresponding presynaptic $\mathrm{Ca}$ transient. A linear regression line was fitted $(r=0.99)$. B, The relationship between the initial slopes of the field EPSP and the amplitude of the corresponding presynaptic Ca transients plotted on a linear scale. $C$, Data of $B$ plotted on a logarithmic scale. The line is from Equation 3 with $n=3.5$. D, The relationship between the slopes of the field EPSP and the corresponding Ca influx plotted on a linear scale. $E$, Data of $D$ plotted on a logarithmic scale. The line is from Equation 4 with $m=3.7$.

an increase of the presynaptic Ca transient could account for the maintenance of LTP.

\section{$P P F$ and presynaptic $\mathrm{Ca}$}

Simultaneous measurements of the $\mathrm{Ca}$ transient, the $\mathrm{Ca}$ influx, and the field EPSP were made during paired-pulse stimulation of the SCC pathway with varying interstimulus intervals. Figure $6 \mathrm{~A}$ shows the results of a typical experiment. The relationship between the normalized facilitation of the second field EPSP and the [Ca $]_{\text {ris }}$ immediately before the second stimulation is well fitted by a linear regression line ( $r=0.88$, eight slices; Fig. $6 B$ ). A similar linear relationship between posttetanic potentiation and $[\mathrm{Ca}]_{\text {res }}$ has been observed in crayfish neuromuscular junction (Delaney et al., 1989) and in mossy fiber terminals of the hippocampus (Regehr and Tank, 1991b). The Ca transient and Ca influx evoked by the second stimuli were decreased with shorter interstimulus intervals (Fig. $6 C$; see also Fig. $6 A$, upper and middle traces). The magnitude of the second Ca transient was determined by numerical subtraction of the Ca transient evoked by only the first stimulus from that by paired-pulse stimulus. With an interstimulus interval of $50 \mathrm{msec}$ the normalized magnitude of the second Ca transient was $75 \pm 9 \%$ (four slices). The magnitude of the second Ca transient "recovered" when the interstimulus interval was longer than $300 \mathrm{msec}$ (Fig. 6 A,C). Qualitatively similar results have been observed in the same preparation in which fura-2 AM was nonselectively loaded by incubation and the synaptic transmission was blocked by either CNQX (Saggau and Sheridan, 1989) or kynurenic acid (Hess and Kuhnt, 1992). The decrease of the second Ca transient during a paired-pulse stimulation is unlikely to be due to the saturation of $\mathrm{Ca}$ indicator, because high-frequency stimulation (HFS; $100 \mathrm{~Hz}, 1 \mathrm{sec}$ ) via the SCC pathway caused a much larger increase of Ca level (e.g., from about $50 \mathrm{~nm}$ to $200 \mathrm{~nm}$; data not shown).

\section{Presynaptic Ca transients during LTP}

After obtaining a stable presynaptic Ca transient, Ca influx, and field EPSP for $20 \mathrm{~min}$, LTP was induced by $\mathrm{HFS}(100 \mathrm{~Hz}, 1$ sec) with the same intensity as used in control stimulation given to the SCC pathway. A typical experiment is shown in Figure $7 A$. While about a $60 \%$ potentiation of the initial slope of the field EPSP was measured $30 \mathrm{~min}$ after the HFS (Fig. $7 A$, lower traces), it was not accompanied by any detectable change of the time course or amplitude of either the presynaptic $\mathrm{Ca}$ transient (Fig. $7 A$, upper traces) or $\mathrm{Ca}$ influx (Fig. $7 A$, middle traces). There was no change in the afferent volleys of stimulated SCC axons, which can be regarded as a control for constant stimulus condition. The results from nine experiments are summarized in Figure $7 B$. Following HFS a 50-60\% increase of the slope of the field EPSPs was observed. Despite this pronounced expression of LTP, neither the presynaptic Ca transient nor Ca influx was significantly increased $30 \mathrm{~min}$ after the HFS as compared 
Figure 6. Paired-pulse facilitation. $A$, Original recordings from a typical experiment. Five paired-pulse responses with various interstimulus intervals are shown superimposed. Upper traces, Presynaptic $\mathrm{Ca}$ transients. The amplitude of the Ca transient evoked by the second stimulus was decreased with shorter interstimulus intervals. Middle traces, The corresponding $\mathrm{Ca}$ influx $(d[\mathrm{Ca}] / d t)$ with a time interval $(d t)$ of 2 msec. The amplitude of $\mathrm{Ca}$ influx in response to the second stimulation was decreased with shorter interstimulus intervals. Lower traces, Field EPSPs. PPF was increased as the interstimulus intervals were shortened. $B$, Relationship between PPF and residual presynaptic $\mathrm{Ca}\left([\mathrm{Ca}]_{\mathrm{res}}\right)$. Data were pooled from eight experiments. PPF was measured as the normalized ratio between the slopes of the two field EPSPs. [Ca] res was measured as the Ca concentration at the time the second stimulus was given minus the resting level. $[\mathrm{Ca}]_{\text {res }}$ was normalized to the amplitude of the $\mathrm{Ca}$ transient evoked by the first stimulus. A linear regression line was fitted $(r=$ 0.88). $C$, The amplitudes of the second $\mathrm{Ca}$ transients decreased as the interstimulus interval was shortened. Data pooled from five experiments (error bars are SEM). The magnitude of the second $\mathrm{Ca}$ transient was determined by numerical subtraction of the Ca transient evoked by the first stimulus from the paired response, and was normalized.
A
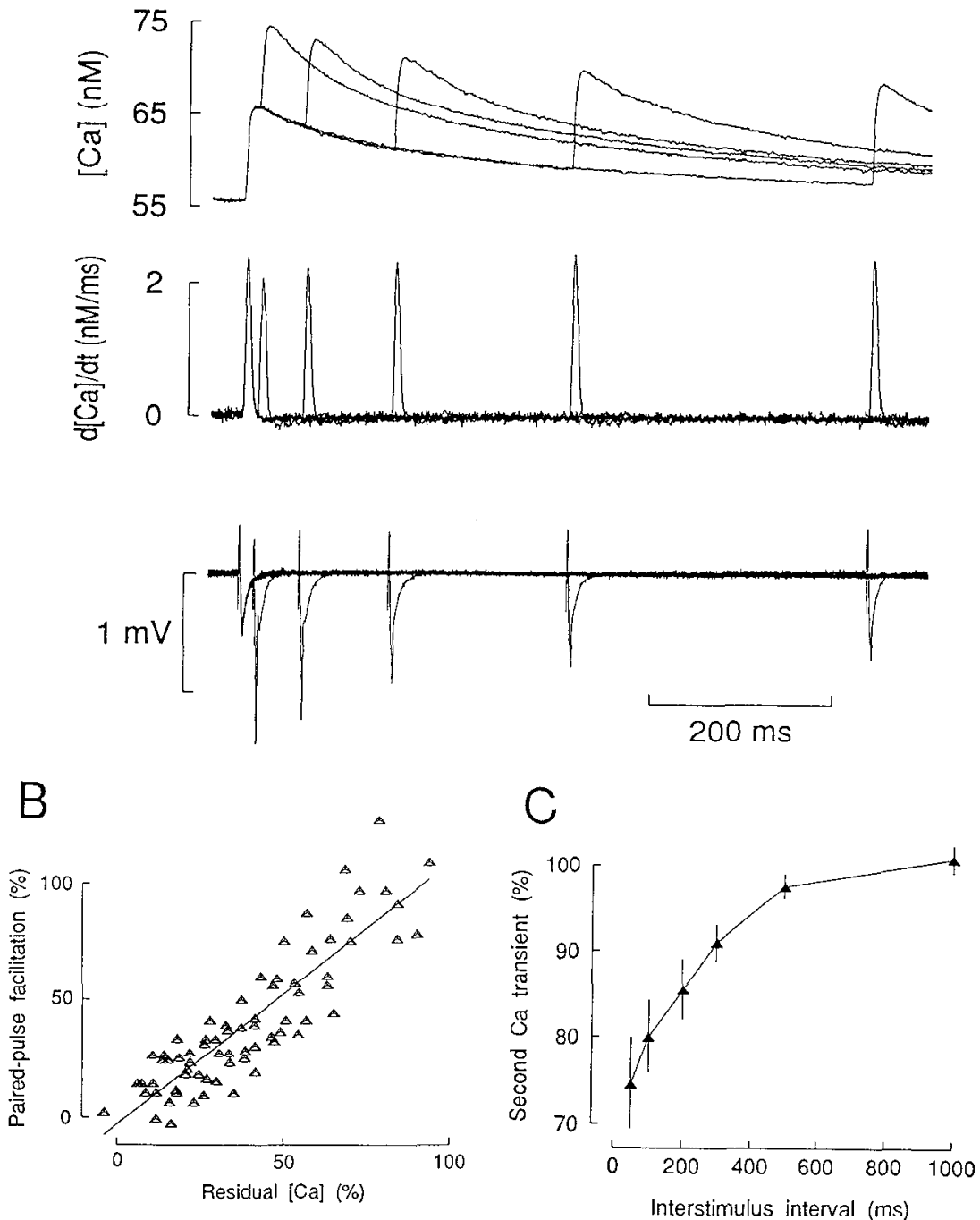

to controls (nine slices; paired $t$ test, $p>0.05$ ). There was a temporary decrease of both the $\mathrm{Ca}$ transient and the $\mathrm{Ca}$ influx about 1 min after the HFS (Fig. $7 B$, upper panels).

The variability of the amplitude of the presynaptic Ca transient before HFS was very small (Fig. $7 B$, upper panel, four measurements before HFS): (1) the maximal variability for a single experiment was $\pm 2.3 \%$ (SEM), and (2) the pooled mean from nine experiments was $100 \pm 1.4 \%$ (mean \pm SEM) with a $95 \%$ upper contidence limit of $104.5 \%$. Based on the power relationship between the field EPSP and both the presynaptic $\mathrm{Ca}$ transient and the $\mathrm{Ca}$ influx described in Equations 3 and 4, a 50\% increase of the slope of the field EPSP would correspond to an increase of the Ca transient to about $112 \%$, which is well above the $95 \%$ upper confidence limit of the pooled mean before HFS. This demonstrates that our $\mathrm{Ca}$ measurements are sufficiently sensitive to detect a possible change of the Ca transient, if a substantial fraction of LTP would be expressed by an increase of this presynaptic Ca transient.

\section{Presynaptic residual Ca levels during LTP}

Having ruled out the possibility that an increase of the presynaptic Ca transient may contribute to the expression of LTP, we explored a second possibility, namely, that the $[\mathrm{Ca}]_{\text {res }}$ contributes to the maintenance of LTP. Following $20 \mathrm{~min}$ of stable recording of both the field EPSP and the resting Ca level, LTP was induced by HFS. After the HFS a transient increase $(5-10 \mathrm{~min})$ of $[\mathrm{Ca}]_{\text {res }}$ was observed, the time course of which (Fig. 8, top) was similar to that of short-term potentiation of field EPSP (Fig. 8, bottom). However, the expression of LTP (50-60\% potentiation of the slope of the field EPSP, nine slices) did not result in any significant sustained [Ca $]_{\text {res }}$ (Fig. 8). We computed an expected [Ca $]_{\text {res }}$ following HFS based on both the relationship between PPF and $[\mathrm{Ca}]_{\text {res }}$, determined before the induction of LTP in each experiment (compare Fig. 6B), and the assumption that sustained increase of $[\mathrm{Ca}]_{\text {res }}$ may account for the maintenance of LTP. The clear difference in the expected and the measured $[\mathrm{Ca}]_{\text {res }}$ (Fig. 8, top) further confirmed that the maintenance of LTP is not due to a sustained [Ca $]_{\text {res. }}$. A similar observation has been made in mossy fiber terminals (Regehr and Tank, 1991b). Based on the relationship between PPF and residual $\mathrm{Ca}$ level, we estimate that the transient increase of residual Ca level may account for at most $30 \%$ of the potentiation, while the maximum potentiation of the slope of field EPSP after HFS was about $250 \%$ of controls. 
A

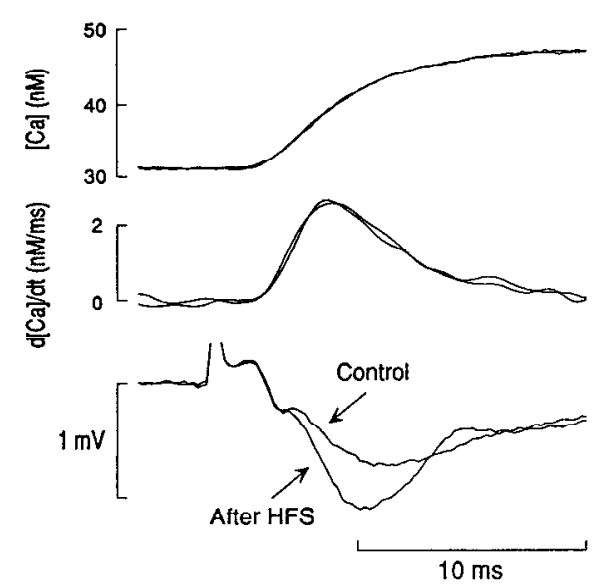

B

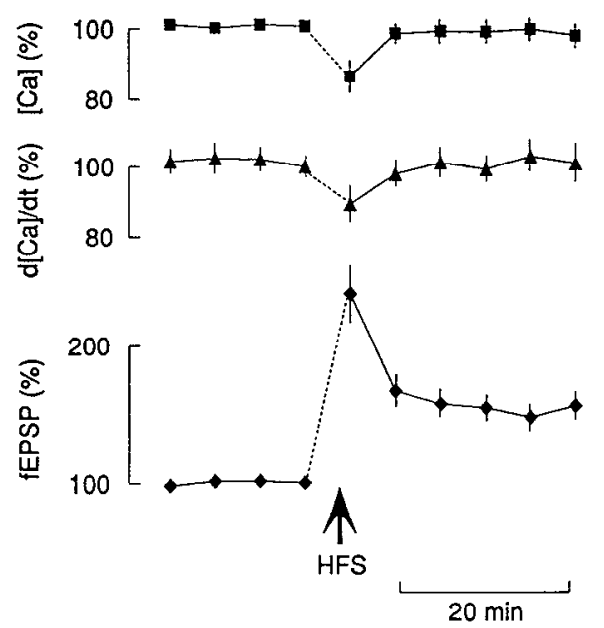

Figure 7. No change of the presynaptic Ca transient or the corresponding Ca influx during the maintenance of LTP. $A$, Calcium signals and field EPSPs were recorded simultaneously and representative responses from a typical experiment are shown superimposed. HFS (100 Hz, 1 sec) resulted in about a $60 \%$ increase of the initial slope of the field EPSP, 30 min after HFS (lower traces), whereas both the presynaptic calcium transients (upper traces) and $\mathrm{Ca}$ influx (middle traces) did not reveal any detectable change. $B$, The normalized pooled data from nine experiments (error bars are SEM; note that some error bars are smaller than their symbols). HFS induced a persistent increase of the slopes of field EPSPs ( $f E P S P$; $50-$ $60 \%$ ), following a short period of posttetanic potentiation (lower panel). The upper and middle panels demonstrate, after a short posttetanic period of reduction, no significant increase in the amplitude of presynaptic Ca transients ([Ca]) and Ca influx ( $d[\mathrm{Ca} / d t)$, respectively. The amplitude of the presynaptic $\mathrm{Ca}$ transient was measured as the difference between the peak of a transient and the level before the stimulus.

\section{Discussion}

We have demonstrated the practicality of simultaneously monitoring the presynaptic Ca transient while recording the field EPSP evoked by a single stimulus at CA3-CA1 synapses of the hippocampal slice. Our data show that the slope of field EPSP varies approximately as the fourth power of the amplitude of both the presynaptic $\mathrm{Ca}$ transient and the $\mathrm{Ca}$ influx, that PPF is linearly related to $[\mathrm{Ca}]_{\text {res }}$, and that LTP is not associated with any increase of presynaptic $\mathrm{Ca}$ influx or sustained $[\mathrm{Ca}]_{\mathrm{res}}$.

\section{Ca indicator loading technique}

Our injection loading technique (Saggau et al., 1992) shares the same principle as the localized perfusion loading method developed by Regehr and Tank (1991a). However, it provides several advantages: (1) the use of only onc pipctte for injection without the need for an additional sewer pipette, (2) the injection of only a small amount of fura- 2 AM solution into the slice with less risks of contamination and without the need for a period of extended local perfusion ( $30 \mathrm{~min}$ ), (3) the possibility of loading structures that are deeper from the surface without the need for turning over the slices, and (4) the potential of loading a $\mathrm{Ca}$ indicator into nonsuperficial structures in intact neural tissues. Employing a single photodiode, the injection loading technique provides a sufficient signal-to-noise ratio for measuring not only the $[\mathrm{Ca}]_{\text {res }}$, but also the presynaptic $\mathrm{Ca}$ transient evoked by a single stimulus. The recorded Ca signals showed high signal-tonoise ratio at high temporal resolution. The simultaneous recording of both the presynaptic $\mathrm{Ca}$ transient and the field EPSP was shown to be very stable over a long period of time. This suggests that this technique could be used to study the modulation of presynaptic Ca channels by various transmitters and $\mathrm{Ca}$ channel blockers that are thought to affect presynaptic $\mathrm{Ca}$ currents, but have been difficult to test so far. As we have dem- onstrated, the injection loading technique can be applied not only to presynaptic terminals but also to postsynaptic dendrites. Loading dendrites with a $\mathrm{Ca}$ indicator provides a way to study the receptor-gated and voltage-gated $\mathrm{Ca}$ influx into these postsynaptic structures evoked by a single synaptic or antidromic activation.

The presynaptic $\mathrm{Ca}$ accumulation evoked by tetanic stimulation at the crayfish neuromuscular junction has been shown to occur locally in boutons and small processes where synaptic vesicles and synaptic "active zones" are located (Zucker et al., 1991). Our data show that the synaptic transmission is nonlinearly related to both the $\mathrm{Ca}$ transient and the $\mathrm{Ca}$ influx (Figs. $4,5)$. We have shown that the N-type Ca channel blocker $\omega$-conotoxin partially blocks both the presynaptic $\mathrm{Ca}$ transient and the field EPSP (Wu and Saggau, 1992), which is consistent with the finding that depolarization induced $\mathrm{Ca}$ influx in brain synaptosomes is partially blocked by $\omega$-conotoxin (Reynolds et al., 1986; Pocock et al., 1992). Taken together, these findings suggest that the evoked presynaptic Ca signals we recorded were primarily from the presynaptic terminals where the transmitter was released, although the optically recorded area includes presynaptic fibers. We did not see any significant change of the field EPSP after injection of fura-2 AM, and the magnitudes of PPF and LTP are similar to other studies (e.g., Malenka et al., 1988; Muller and Lynch, 1989). This suggests that injection of fura-2 $\mathrm{AM}$ and illumination with short-wavelength light do not affect the normal physiology of the investigated nerve terminals. It has been reported that the time course of $\mathrm{Ca}$ decay is substantially lengthened with higher concentration of fura-2 (Regehr and Tank, 1992). The half-decay time (238 $\pm 18 \mathrm{msec}$, eight slices) of the $\mathrm{Ca}$ transient evoked by a single stimulus shows only a small deviation in each of our experiments, suggesting that the fura- 2 concentration inside the terminals was similar from experiment to experiment. 

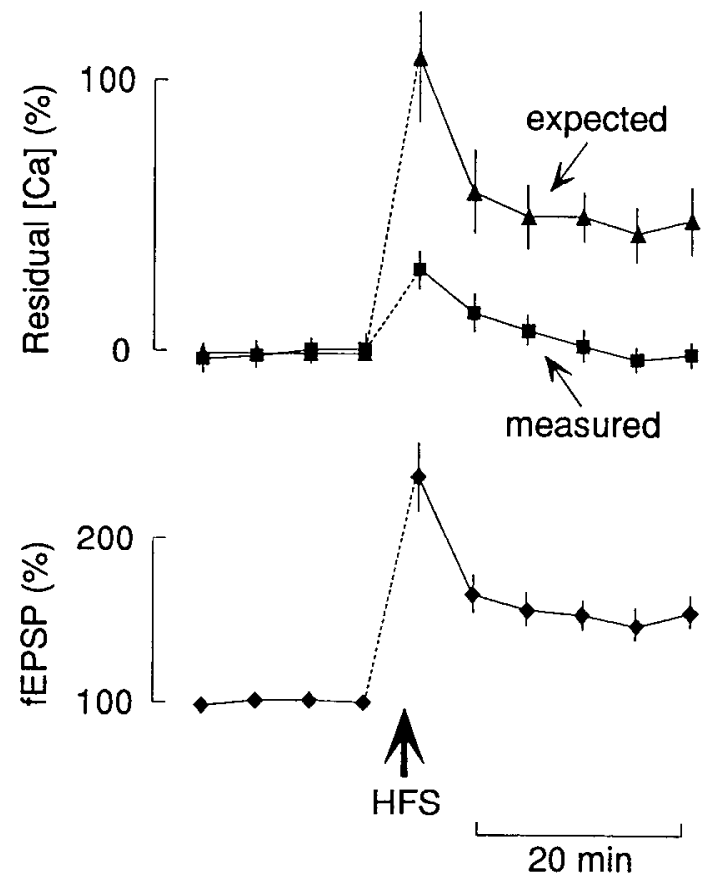

Figure 8. A persistent increase of presynaptic $[\mathrm{Ca}]_{\text {res }}$ is not detected during the expression of LTP. Data are normalized ( $n=9$ slices, error bars are SEM). Lower panel, The slope of field EPSP ( $f E P S P)$ was potentiated by more than $50 \%$ after HFS $(100 \mathrm{~Hz}, 1 \mathrm{sec})$. Upper panel, The measured $[\mathrm{Ca}]_{\mathrm{res}}$ following an HFS showed only a clear transient increase, but no significant persistent increase; the expected $[\mathrm{Ca}]_{\mathrm{res}}$ was calculated from the slope of field EPSPs (lower panel) by applying their individual calibration curves of Figure $6 B$, under the assumption that the $[\mathrm{Ca}]_{\text {res }}$, like PPF, does account for the maintenance of LTP.

\section{Relationship between synaptic transmission, the presynaptic Ca transient, and Ca influx}

Augustine et al. (1985a) showed that the integral over time of the presynaptic $\mathrm{Ca}$ current in the squid giant synapse corresponds excellently to absorption signals obtained with Arsenazo III, another Ca indicator. Our Ca signals showed very little noise, which allowed us to take the reverse approach and derive the $\mathrm{Ca}$ transient to obtain a volume average $\mathrm{Ca}$ influx, which is presumably proportional to the average presynaptic Ca current. Although the duration of the field EPSP was much shorter than the recorded Ca transient, which represents the mean free cytoplasmic $\mathrm{Ca}$ concentration in the activated presynaptic terminals, the duration of the $\mathrm{Ca}$ influx was close to that of the field EPSP. The peak of the Ca influx corresponds to the moment when the slope of the field EPSP is maximal, which is about $2.1 \mathrm{msec}$ before the peak of the field EPSP (see Fig. $3 B$ ). Therefore, the time course of the $\mathrm{Ca}$ influx may closely reflect the period of transmitter release evoked by a single stimulation of the SCC pathway.

Our data show that the $\mathrm{Ca}$ influx is to a great extent linearly related to the presynaptic $\mathrm{Ca}$ transient. This suggests that the amplitude of a presynaptic $\mathrm{Ca}$ transient may well reflect the presynaptic $\mathrm{Ca}$ influx, and hence the average presynaptic $\mathrm{Ca}$ current. Our finding that the slope of the field EPSP is a power function of $\mathrm{Ca}$ influx is in agrecment with previous cxperiments performed in the squid giant synapse (Augustine et al., 1985b; Augustine and Charlton, 1986). The power relationship between the slope of the field EPSP and the amplitude of the Ca transient in our experiments is consistent with the finding that the EPSP amplitude is nonlinearly related to the presynaptic $\mathrm{Ca}$ accumulation induced by tetanic stimulation at crayfish neuromuscular junction (Zucker et al., 1991).

Recently, several studies have demonstrated sharp spatial $\mathrm{Ca}$ gradients in presynaptic terminals during synaptic transmission (Swandulla et al., 1991; Llinás et al., 1992). Fura-2 signals provide a measure of the average $\mathrm{Ca}$ concentration across the diameter of the terminal, and as such may greatly underestimate the $\mathrm{Ca}$ concentration near the inner surface of the membrane, where $\mathrm{Ca}$ is entering during and immediately after an action potential invades the terminal. Since the average $\mathrm{Ca}$ level may not be linearly related to the local $\mathrm{Ca}$ level, the power relationship we measured between the field EPSP and the presynaptic Ca transient may not well reflect the relationship between the transmitter release and the local $\mathrm{Ca}$ level responsible for this release. Nevertheless, the measured power relationship can be used to estimate reliably whether application of a drug or any other manipulation modifies synaptic transmission by modulating the presynaptic $\mathrm{Ca}$ influx.

\section{$P P F$ and presynaptic $\mathrm{Ca}$}

To our knowledge, this is the first time that the relationship between PPF and residual Ca level has been measured in the mammalian nervous system. As discussed above, the spatial distribution of $\mathrm{Ca}$ across the terminal following an action potential may complicate the measurement of $[\mathrm{Ca}]_{\text {res }}$ for PPF. However, it has been suggested that equilibration of $\mathrm{Ca}$ concentration inside a terminal at the crayfish neuromuscular junction (Delaney et al., 1989) and in a $5 \mu \mathrm{m}$ spherical model terminal for simulation of Ca diffusion (Smith and Zucker, 1980) is substantially complete within $100 \mathrm{msec}$ after an action potential. This suggests that most of our measurements of $[\mathrm{Ca}]_{\text {res }}$ for PPF with interstimulus intervals of $100 \mathrm{msec}$ or more were made at a time when the Ca concentration had basically equilibrated. The very fast "on" rate constant $\left(k_{+1}\right)$ for Ca-fura-2 reaction allows fura-2 to track the rapid change of $\mathrm{Ca}$ concentration during the rising phase of a $\mathrm{Ca}$ transient (Kao and Tsien, 1988). However, the slower "off" rate constant $\left(k_{-1}\right)$ may limit the ability of fura- 2 to track faithfully rapid changes in Ca concentration during the falling phase of a Ca transient. It has been estimated that $k_{-1}$ is about $97 \mathrm{sec}^{-1}$ in vitro (Kao and Tsien, 1988), and $23 \mathrm{sec}^{-1}$ in myoplasm (Baylor and Hollingworth, 1988). Assuming that $\mathrm{k}_{-1}$ for Ca-fura-2 reaction in the presynaptic terminals of CA3-CA1 synapses is within 23-97 $\mathrm{sec}^{-1}$, this implies that the $[\mathrm{Ca}]_{\text {res }}$ estimated with fura- 2 should track true $[\mathrm{Ca}]_{\text {res }}$ with a delay of no more than about $10-40 \mathrm{msec}$. Since we measured $[\mathrm{Ca}]_{\mathrm{rcs}}$ over a range of $1 \mathrm{sec}$ following the single stimulus, a delay of less than about $10-40 \mathrm{msec}$ in estimated $[\mathrm{Ca}]_{\text {res }}$ should not affect our basic conclusion.

The approximately linear relationship between PPF and $[\mathrm{Ca}]_{\text {res }}$ shown in Figure $6 B$ is consistent with the findings that posttetanic potentiation is approximately linearly related to the presynaptic $[\mathrm{Ca}]_{\text {res }}$ in both crayfish neuromuscular junction (Delaney et al., 1989; Delaney and Tank, 1991) and mossy fiber terminals of the hippocampus (Regehr and Tank, 1991b). However, PPF was accompanied by a decrease of the second Ca transient or the $\mathrm{Ca}$ influx when the interstimulus interval was less than $300 \mathrm{mscc}$. This is in agrecment with carlicr investigations performed in the hippocampus by Saggau and Sheridan (1989) and Hess and Kuhnt (1992), but is in contrast to the finding that two equal $\mathrm{Ca}$ transients were elicited by pairedpulse stimulation in squid giant presynaptic terminals (Charlton 
et al., 1982). Taking this into account and calculating the expected facilitation by applying the power relationship between the field EPSP and the Ca transient, one might even expect a nonlinear relationship between $\mathrm{PPF}$ and $[\mathrm{Ca}]_{\text {res }}$. Interestingly, synaptic augmentation was found to be nonlincarly related to $[\mathrm{Ca}]_{\text {res }}$ in the squid giant synapse (Swandulla et al., 1991), in which PPF was not accompanied by a decrease of the second Ca transient (Charlton et al., 1982).

The mechanism of decrease of the second $\mathrm{Ca}$ transient in response to paired-pulse stimulation is unknown. It is unlikely to be due to the saturation of fura-2, because HFS could cause a much larger increase of $\mathrm{Ca}$ level. Three likely mechanisms may account for this phenomenon: (1) Ca- and/or voltage-dependent inactivation of presynaptic $\mathrm{Ca}$ channels (Fisher et al., 1990; Kay, 1991); (2) transmitters or modulators such as glutamate and adenosine released from synaptic vesicles in response to the first stimulus may activate presynaptic autoreceptors, which in turn couple to presynaptic Ca channels and cause an inactivation of these Ca channels (Baskys and Malenka, 1991; Thompson et al., 1992); and (3) a Ca- and/or voltagedependent residual activation of potassium channels might in principle shunt the second spike. In general, the effect of a decreased second $\mathrm{Ca}$ transient or the $\mathrm{Ca}$ influx on reducing transmitter release at the second pulse may be masked by the facilitating effect of a residual $\mathrm{Ca}$ level set by the first pulse. The reduction of both the $\mathrm{Ca}$ transient and $\mathrm{Ca}$ influx during a short period after an HFS (Fig. 8) might also be explained by the above considerations.

\section{Possible mechanisms for the maintenance of LTP}

Within the resolution of the methods employed, our results directly demonstrate in the CA3-CA1 synapses of the guinea pig hippocampus that expression of LTP is independent of any detectable persistent increase of the presynaptic $\mathrm{Ca}$ transient or residual Ca level. The LTP-inducing HFS is followed by a shortterm $[\mathrm{Ca}]_{\mathrm{res}}$ (about 5-10 $\mathrm{min}$ ), which may contribute to shortterm potentiation. An earlier study showed a differential effect of changing $\mathrm{Ca}$ concentrations versus LTP induction on PPF, which indirectly suggests that presynaptic Ca currents are not responsible for LTP in area CAl of hippocampus (Muller and Lynch, 1989). Our results agree with this suggestion. The results of another recent study are in accordance with our findings: in cultured CA3-CA1 neurons a sustained potentiation of the frequency of miniature synaptic currents following the application of the excitatory neurotransmitter glutamate was demonstrated in the absence of Ca entry into the presynaptic terminal (Malgaroli and Tsien, 1992). In addition, at mossy fiber-CA3 synapses, although being different from the investigated CA3-CA1 synapses in that the induction of LTP is NMDA receptor independent (for review, see Johnston et al., 1992), the maintenance of LTP has been reported to be independent of presynaptic residual $\mathrm{Ca}$ (Regehr and Tank, 1991b). These findings together with our results raise the question of possible common presynaptic mechanisms of expression and maintenance along with distinct postsynaptic mechanisms of LTP induction in different synapses of mammalian CNS.

Since our study was restricted to the presynaptic site, possible changes in postsynaptic receptor sensitivity were not investigated; thus, our results do not exclude a postsynaptic contribution to the maintenance of LTP. Two recent investigations carried out at the CA3-CA1 synapses proposed concurrent preand postsynaptic modification as judged by an increase of quan- tal content and quantal amplitude (Kullmann and Nicoll, 1992; Liao et al., 1992). In both studies, the relative contribution of quantal content and quantal amplitude during LIP varied significantly among different experiments. The increase in these quantal parameters during LTP was inverscly correlated to their initial values (Liao et al., 1992). Since we did not measure quantal parameters, we cannot judge the relative contribution of presynaptic and postsynaptic enhancement during LTP in our experiments. However, accumulated evidence has shown that a substantial component of LTP can be accounted for by presynaptic enhancement in CA3-CA1 synapses (Bekkers and Stevens, 1990; Malinow and Tsien, 1990; Kullmann and Nicoll, 1992; Liao et al., 1992; Malgaroli and Tsien, 1992). Given such a presynaptic contribution to the maintenance of LTP, our results suggest that some intermediate step between the presynaptic $\mathrm{Ca}$ transient and transmitter release is the likely site of persistent change. A likely site for possible retrograde messengers may be the intracellular $\mathrm{Ca}$ receptor mediating docking and fusion of synaptic vesicles.

\section{References}

Andersen P, Silfvenius H, Sundberg SH, Sveen O, Wigstrom H (1978) Functional characteristics of unmyelinated fibers in the hippocampal cortex. Brain Res 144:11-18.

Augustine GJ, Charlton MP (1986) Calcium dependence of presynaptic calcium current and post-synaptic response at the squid giant synapse. J Physiol (Lond) 381:619-640.

Augustine G.I, Eckert R (1984) Divalent cations differentially support transmitter release at the squid giant synapse. J Physiol (Lond) 346: 257-271.

Augustine GJ, Charlton MP, Smith SJ (1985a) Calcium entry into voltage-clamped presynaptic terminals of squid. J Physiol (Lond) 367 : 143-162.

Augustine GJ, Charlton MP, Smith SJ (1985b) Calcium entry and transmitter release at voltage-clamped nerve terminals of squid. $\mathbf{J}$ Physiol (Lond) 369:163-181

Baskys A, Malenka RC (1991) Agonists at metabotropic glutamate receptors presynaptically inhibit EPSCs in neonatal rat hippocampus. J Physiol (Lond) 444:687-701.

Baylor SM, Hollingworth S (1988) Fura-2 calcium transients in frog skeletal muscle fibres. J Physiol (Lond) 403:151-192.

Bekkers IM, Stevens CF (1990) Presynaptic mechanism for long-term potentiation in the hippocampus. Nature 346:724-729.

Byrne JH, Cleary LJ, Baxter DA (1989) Aspects of the neural and molecular mechanisms of short-term sensitization in Aplysia: modulatory effects of serotonin and cAMP on duration of action potentials, excitability and membrane currents in tail sensory neurons. In: The biology of memory (Squire LR, Lindenlaub E, eds), pp 7-31. Stuttgart: Schattauer.

Charlton MP, Smith SJ, Zucker RS (1982) Role of presynaptic calcium ions and channels in synaptic facilitation and depression at the squid giant synapse. J Physiol (Lond) 323:173-193.

Connor JA, Kretz R, Shapiro E (1986) Calcium levels measured in a presynaptic neurone of Aplysia under conditions that modulate transmitter release. J Physiol (Lond) 375:625-642.

Delaney KR, Tank DW (1991) Calcium-dependent and calcium-independent enhancement of transmitter release at the crayfish neuromuscular junction studied with fura-2 imaging. Ann NY Acad Sci 635:452-455.

Delaney KR, Zucker RS, Tank DW (1989) Calcium in motor nerve terminals associated with posttetanic potentiation. J Neurosci 9:35583567.

Delaney KR, Tank DW, Zucker RS (1991) Presynaptic calcium and serotonin-mediated enhancement of transmitter release at crayfish neuromuscular junction. J Neurosci 11:2631-2643.

Dingledine R, Somjen G (1981) Calcium dependance of synaptic transmission in the hippocampal slice. Brain Res 207:218-222.

Dodge FA, Rahamimoff R (1967) Co-operative action of calcium ions in transmitter release at the neuromuscular junction. J Physiol (Lond) 193:419-432. 
Fazeli MS (1992) Synaptic plasticity-on the trail of the retrograde messenger. Trends Neurosci 15:115-117.

Fisher RE, Gray R, Johnston D (1990) Properties and distribution of single voltage-gated calcium channels in adult hippocampal neurons. J Neurophysiol 64:91-103.

Grynkiewiez G, Poenie M, Tsien RY (1985) A new generation of $\mathrm{Ca}^{++}$ indicators with greatly improved fluorescence properties. J Biol Chem $260: 3440-3450$.

Hcss G, Kuhnt U (1992) Presynaptic calcium transients evoked by paired-pulse stimulation in the hippocampal slice. Neuroreport 3:361364.

Johnston D, Williams S, Jaffe DB, Gray R (1992) NMDA-receptorindependent long-term potentiation. Annu Rev Physiol 54:489-505.

Kandel ER, Schwartz JH (1982) Molecular biology of learning: modulation of transmitter release. Science 218:433-443.

Kao JPY, Tsien RY (1988) $\mathrm{Ca}^{2+}$ binding kinetics of fura-2 and Azo-1 from temperature-jump relaxation measurements. Biophys J 53:635639.

Katz B, Miledi R (1968) The role of calcium in neuromuscular facilitation. J Physiol (Lond) 195:48 1-492.

Katz B, Miledi R (1970) Further study of the role of calcium in synaptic transmission. J Physiol (Lond) 207:789-801

Kay AR (1991) Inactivation kinetics of calcium current of acutely dissociated CA1 pyramidal cells of the mature guinea-pig hippocampus. J Physiol (Lond) 437:27-48.

Korn H, Faber DS (1991) Quantal analysis and synaptic efficacy in the CNS. Trends Neurosci 14:439-445.

Kullmann DM, Nicoll RA (1992) Long-term potentiation is associated with increases in quantal content and quantal amplitude. Nature 357: 240-244.

Lev-Ram V, Grinvald A (1987) Activity-dependent calcium transients in central nervous system myelinated axons revealed by the calcium indicator fura-2. Biophys $\mathrm{J}$ 52:571-576.

I iao D, Jones A, Malinow R (1992) Direct measurement of quantal changes underlying long-term potentiation in CA1 hippocampus. Neuron 9:1089-1097.

Llinás R, Steinberg IZ, Walton K (1976) Presynaptic calcium currents and their relation to synaptic transmission: voltage clamp study in squid giant synapse and theoretical model for the calcium gate. Proc Natl Acad Sci USA 73:2918-2922.

Llinás R, Steinberg IZ, Walton K (1981) Relationship between presynaptic calcium current and postsynaptic potential in squid giant synapse. Biophys J 33:323-352.

Llinás R, Sugimori M, Silver RB (1992) Microdomains of high calcium concentration in a presynaptic terminal. Science 256:677-679.

Malenka RC, Kauer JA, Zucker RS, Nicoll RA (1988) Postsynaptic calcium is sufficient potentiation of hippocampal synaptic transmission. Science 242:81-84.

Malgaroli A, Tsien RW (1992) Glutamate-induced long-term potentiation of the frequency of miniature synaptic currents in cultured hippocampal neurons. Nature 357:134-139.
Malinow R, Tsien RW (1990) Presynaptic enhancement shown by whole-cell recordings of long-term potentiation in hippocampal slices. Nature 346:177-180.

Mulkeen D, Anwyl R, Rowan M (1988) The effects of external calcium on long-term potentiation in the rat hippocampal slice. Brain Res 447:234-238

Muller D, Lynch G (1989) Evidence that changes in presynaptic calcium currents are not responsible for long-term potentiation in hippocampus. Brain Res 479:290-299.

Pocock JM, Venema VJ, Adams ME (1992) $\omega$-Agatoxins differentially block calcium channels in locust, chick and rat synaptosomes. Neurochem Int 20:263-270.

Regehr WG, Tank DW (1991a) Selective fura-2 loading of presynaptic terminals and nerve cell processes by local perfusion in mammalian brain slice. J Neurosci Methods 37:111-119.

Regehr WG, Tank DW (1991b) The maintenance of LTP at hippocampal mossy fiber synapses is independent of sustained presynaptic calcium. Neuron 7:451-459.

Regehr WG, Tank DW (1992) Calcium concentration dynamics produced by synaptic activation of CA1 hippocampal pyramidal cells. $J$ Neurosci 12:4202-4223.

Reynolds IJ, Wagner JA, Snyder SH, Thayer SA, Oliver BM, Miller RJ (1986) Brain voltage-sensitive calcium channel subtypes differentiated by $\omega$-conotoxin fraction GVIA. Proc Natl Acad Sci USA 83: 8804-8807.

Saggau P, Sheridan RD (1989) Optical monitoring of presynaptic $\mathrm{Ca}^{2+}$ changes in area $\mathrm{CAl}$ of the hippocampus using the $\mathrm{Ca}^{2+}$-sensitive fluorescent dye, fura-2. Neurosci Lett 36:S54

Saggau P, Wu LG (1992) The expression of long-term potentiation in hippocampal area CAl does not change presynaptic calcium. Soc Neurosci Abstr 18:1459.

Saggau P, Wu LG, Yehezkely I (1992) Optical recording of transients of cytosolic free calcium in synaptic structures of hippocampal CA1 pyramidal cells by employing a novel selective loading technique in brain slices. Biophys J 61:A509.

Smith SJ, Zucker RS (1980) Aequorin response facilitation and intracellular calcium accumulation in molluscan neurones. J Physiol (Lond) 300:167-196.

Swandulla D, Hans M, Zipser K, Augustine GJ (1991) Role of residual calcium in synaptic depression and posttetanic potentiation: fast and slow calcium signaling in nerve terminals. Neuron 7:915-926.

Thompson SM, Haas HL, Gahwiler BH (1992) Comparison of the actions of adenosine at pre- and postsynaptic receptors in the rat hippocampus in vitro. J Physiol (Lond) 451:347-363.

Wu LG, Saggau P (1992) Different voltage-dependent calcium channels at pre- and postsynaptic structures in hippocampal area CAl. Soc Neurosci Abstr 18:429.

Zucker RS, Delaney KR, Mulkey R, Tank DW (1991) Presynaptic calcium in transmitter release and posttetanic potentiation. Ann NY Acad Sci 635:191-207. 